\title{
Almost all trees have an even number of independent sets
}

\author{
Stephan G. Wagner \\ Department of Mathematical Sciences \\ Stellenbosch University \\ Private Bag X1, Matieland 7602, South Africa \\ swagner@sun.ac.za
}

Submitted: Mar 28, 2009; Accepted: Jul 21, 2009; Published: Jul 31, 2009

Mathematics Subject Classifications: 05C30; 05A16, 05C05, 05C69

\begin{abstract}
This paper is devoted to the proof of the surprising fact that almost all trees have an even number of independent vertex subsets (in the sense that the proportion of those trees with an odd number of independent sets tends to 0 as the number of vertices approaches $\infty$ ) and to its generalisation to other moduli: for fixed $m$, the probability that a randomly chosen tree on $n$ vertices has a number of independent subsets that is divisible by $m$ tends to 1 as $n \rightarrow \infty$.
\end{abstract}

\section{Introduction}

The number of independent vertex subsets is one of many interesting graph parameters that have been studied in the past. It was introduced to the mathematical literature in a paper by Prodinger and Tichy in 1982 [21], where it was shown - amongst other things such as explicit formulæ for various classes of graphs - that the star and the path maximize resp. minimize the number of independent sets among all trees of given size. In subsequent works, Kirschenhofer, Prodinger and Tichy [11, 12] investigated the average number of independent sets in certain classes of rooted trees (such as binary trees), providing some nice combinatorial results.

Generally, the number of independent sets is a parameter that is particularly wellstudied for trees and tree-like structures (forests, unicyclic graphs, etc.): a paper of Lin and Lin [15] extends the aforementioned results, and in recent years a wealth of literature on the so-called extremal problem (given a class of graphs, determine those members of the class that maximize or minimize the number of independent sets) has developed; see for instance $[9,14,19,20,23]$ for some nice results in this direction. Part of the interest is due to the fact that this parameter plays a role in mathematical chemistry, where it is 
known as the Merrifield-Simmons index [14, 17, 23]. A problem that arises in this context is the inverse problem: determine a graph within a given class (such as the class of all trees) with a given number of independent sets. It is an open conjecture [13] that all but finitely many positive integers can be represented as the number of independent sets of some tree. A partial result in this direction is due to Linek [16] who was able to show by means of a clever yet elementary construction that every positive integer is the number of independent sets of some bipartite graph.

While there are interesting distribution results for the sizes of independent sets in graphs (see [3]), there is no distribution result known for the number of independent sets itself yet. Considering the number of independent sets of a randomly chosen tree on $n$ vertices as a random variable, does it (suitably normalized) tend to a limiting distribution as $n \rightarrow \infty$ ? Results in this direction are known for many other tree parameters, such as the height or the Wiener index (the sum of all the distances between pairs of vertices; see $[5,10])$.

The present paper provides some explanation why the inverse problem for trees is harder than it might seem to be and why it is not so easy to obtain distribution results of the aforementioned type either. Suprisingly, it turns out the number of independent sets of a randomly selected tree is more likely to be even than to be odd; indeed, the probability that the number is even tends to 1 as the number of vertices approaches $\infty$. Even more generally, for any fixed modulus $m$, the probability that the number of independent sets of a randomly selected tree is divisible by $m$ tends to 1 as the number of vertices increases. There seems to be no obvious intuitive explanation for this phenomenon, considering that it was shown for other parameters (e.g. the number of leaves $[1,4]$ or again the Wiener index [22]) that the number of trees with even parameter value is asymptotically the same as the number of trees with odd parameter value. There is also no particular evidence if one considers small trees or special cases only: for instance, a star on $n$ vertices has exactly $2^{n-1}+1$ independent vertex sets, which is odd for $n>1$. The result is only asymptotic in nature.

More precisely speaking, we will prove the following:

Theorem 1 Let $m>1$ be a fixed integer, let $\mathcal{T}_{n}$ denote the set of all trees on $n$ vertices, and let $\mathcal{T}_{n}^{(m)}$ be the set of all trees with the property that the number of independent sets is divisible by $m$. There exists a constant $c<1$ that only depends on $m$ such that

$$
\frac{\left|\mathcal{T}_{n} \backslash \mathcal{T}_{n}^{(m)}\right|}{\left|\mathcal{T}_{n}\right|}=O\left(c^{n}\right) .
$$

By no means this implies that there are "few" trees with e.g. an odd number of independent sets. Their number still increases exponentially, and we will show in Section 3 that the constant $c$ is not too far away from 1 in the case $m=2$. 


\section{Proof of the main result}

We will make use of a recursive formula for the number $i(T)$ of independent sets of a rooted tree $T$. To this end, we have to introduce an auxiliary parameter $i_{0}(T)$, which denotes the number of independent sets of a rooted tree $T$ that do not contain the root of $T$. If $T_{1}, T_{2}, \ldots, T_{k}$ are the subtrees of $T$ (see Figure 1 ), we have the following formulæ, which are easy to verify:

$$
i(T)=\prod_{j=1}^{k} i\left(T_{j}\right)+\prod_{j=1}^{k} i_{0}\left(T_{j}\right), \quad i_{0}(T)=\prod_{j=1}^{k} i\left(T_{j}\right) .
$$

Now we define subsets $\mathcal{R}_{n}^{(m)}(r, s)$ of the set $\mathcal{R}_{n}$ of all rooted trees on $n$ vertices as follows:

$$
\mathcal{R}_{n}^{(m)}(r, s)=\left\{T \in \mathcal{R}_{n}: i(T) \equiv r \bmod m, i_{0}(T) \equiv s \bmod m\right\} .
$$

Furthermore, we write $\mathcal{R}=\bigcup_{n \in \mathbf{N}} \mathcal{R}_{n}$ and $\mathcal{R}^{(m)}(r, s)=\bigcup_{n \in \mathbf{N}} \mathcal{R}_{n}^{(m)}(r, s)$ for all $r, s$. If $T$ is now a rooted tree with subtrees $T_{1}, T_{2}, \ldots, T_{k}$, and $T_{j} \in \mathcal{T}^{(m)}\left(r_{j}, s_{j}\right)$ for certain $r_{j}$ and $s_{j}$, then it is possible to determine $i(T)$ and $i_{0}(T)$ (modulo $m$ ) from the values of the $r_{j}$ and $s_{j}$ only. In particular, it should be noted that if one of the subtrees is an element of $\mathcal{R}^{(m)}(0,0)$, then so is $T$.

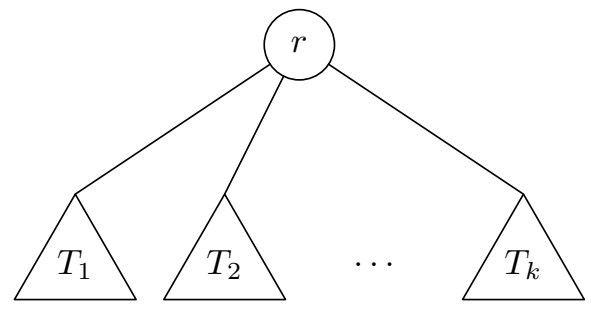

Figure 1: A rooted tree and its subtrees.

Next we need the following lemma:

Lemma 2 For any integer $m>1$, the set $\mathcal{R}^{(m)}(0,0)$ is nonempty.

Proof: We make use of the simple fact (see [21]) that $i\left(P_{n}\right)=F_{n+2}$, where $P_{n}$ is the path on $n$ vertices and $F_{k}$ denotes the $k$-th Fibonacci number $\left(F_{0}=0, F_{1}=1, F_{n}=F_{n-1}+F_{n-2}\right)$. It is well known that for any positive integer $m$, there exists a Fibonacci number (other than 0 ) that is divisible by $m$ (since the sequence of Fibonacci numbers is periodic modulo $m$ and contains the element $\left.F_{0}=0\right)$. Furthermore, if we consider a path $P_{n}$ as a rooted tree, where the root is one of its ends, then we have $i_{0}\left(P_{n}\right)=i\left(P_{n-1}\right)=F_{n+1}$. Now consider the rooted tree $T$ whose subtrees are a path $P_{n}$ and a path $P_{n-1}$, both rooted at one of the ends (the tree $T$ is thus again a path), where $n$ is chosen in such a way that $F_{n+1}$ is divisible by $m$. Then we obtain immediately that

$$
i(T)=F_{n+2} F_{n+1}+F_{n+1} F_{n}=F_{n+1}\left(F_{n+2}+F_{n}\right) \equiv 0 \bmod m
$$


and

$$
i_{0}(T)=F_{n+2} F_{n+1} \equiv 0 \bmod m .
$$

Hence, $T \in \mathcal{R}^{(m)}(0,0)$, which completes the proof.

The rest of the proof of Theorem 1 makes use of generating functions. Let $T(z)$ denote the generating function of $\mathcal{R}$, i.e.

$$
T(z)=\sum_{n \geq 1}\left|\mathcal{R}_{n}\right| z^{n}
$$

and let $U(z)=U^{(m)}(z)$ be the generating function of $\mathcal{R} \backslash \mathcal{R}^{(m)}(0,0)$ (in the following, we always drop the superscript for convenience). It is well known [7] that $T(z)$ satisfies the functional equation

$$
T(z)=z \exp \left(\sum_{k=1}^{\infty} \frac{1}{k} T\left(z^{k}\right)\right) .
$$

The second factor is the generating function for multisets of rooted trees (see [6]): the subtrees of a rooted tree form such a multiset. Since all subtrees of a rooted tree $T \in$ $\mathcal{R} \backslash \mathcal{R}^{(m)}(0,0)$ are again elements of $\mathcal{R} \backslash \mathcal{R}^{(m)}(0,0)$, we have

$$
U(z) \unlhd z \exp \left(\sum_{k=1}^{\infty} \frac{1}{k} U\left(z^{k}\right)\right) .
$$

Here and in the following, we use the notation $A(z) \unlhd B(z)$ if $\left[z^{n}\right] A(z) \leq\left[z^{n}\right] B(z)$ for all $n$, i.e. if the coefficients of $A$ are less than or equal to the respective coefficients of $B$.

Let us now define the power series $\tilde{U}(z)$ by the functional equation

$$
\tilde{U}(z)=z \exp \left(\tilde{U}(z)+\sum_{k=2}^{\infty} \frac{1}{k} U\left(z^{k}\right)\right) .
$$

Then $U(z) \unlhd \tilde{U}(z)$ holds, which is easily proved by induction:

- $\left[z^{1}\right] U(z) \leq 1=\left[z^{1}\right] \tilde{U}(z)$ holds trivially.

- Suppose $\left[z^{k}\right] U(z) \leq\left[z^{k}\right] \tilde{U}(z)$ holds for all $k<n$. Write $U_{n}(z)$ for the truncated series $\sum_{k=1}^{n-1}\left(\left[z^{k}\right] U(z)\right) z^{k}$ and analogously $\tilde{U}_{n}(z)=\sum_{k=1}^{n-1}\left(\left[z^{k}\right] \tilde{U}(z)\right) z^{k}$. Then we have

$$
\begin{aligned}
{\left[z^{n}\right] U(z) } & \leq\left[z^{n}\right] z \exp \left(\sum_{k=1}^{\infty} \frac{1}{k} U\left(z^{k}\right)\right)=\left[z^{n}\right] z \exp \left(U(z)+\sum_{k=2}^{\infty} \frac{1}{k} U\left(z^{k}\right)\right) \\
& =\left[z^{n}\right] z \exp \left(U_{n}(z)+\sum_{k=2}^{\infty} \frac{1}{k} U\left(z^{k}\right)\right) \leq\left[z^{n}\right] z \exp \left(\tilde{U}_{n}(z)+\sum_{k=2}^{\infty} \frac{1}{k} U\left(z^{k}\right)\right) \\
& =\left[z^{n}\right] z \exp \left(\tilde{U}(z)+\sum_{k=2}^{\infty} \frac{1}{k} U\left(z^{k}\right)\right)=\left[z^{n}\right] \tilde{U}(z) .
\end{aligned}
$$


Now we consider the analytic properties of $T(z)$ and $\tilde{U}(z)$. Write

$$
H_{1}(z)=\sum_{k=2}^{\infty} \frac{1}{k} T\left(z^{k}\right) \quad \text { and } \quad H_{2}(z)=\sum_{k=2}^{\infty} \frac{1}{k} U\left(z^{k}\right) .
$$

Then we have

$$
T(z)=z \exp \left(T(z)+H_{1}(z)\right) \quad \text { and } \quad \tilde{U}(z)=z \exp \left(\tilde{U}(z)+H_{2}(z)\right)
$$

which can be rewritten as

$$
-T(z) e^{-T(z)}=-z \exp \left(H_{1}(z)\right) \text { and } \quad-\tilde{U}(z) e^{-\tilde{U}(z)}=-z \exp \left(H_{2}(z)\right) .
$$

Hence the following formulas for $T(z)$ and $\tilde{U}(z)$ hold:

$$
T(z)=-W\left(-z \exp \left(H_{1}(z)\right)\right) \quad \text { and } \quad \tilde{U}(z)=-W\left(-z \exp \left(H_{2}(z)\right)\right)
$$

where $W$ denotes the Lambert $W$ function [2] which is defined implicitly by $z=W e^{W}$. $W$ is analytic except for a branch cut along the negative real axis that ends at $-\frac{1}{e}$. $H_{1}$ and $H_{2}$ have larger radius of convergence than $T$ and $\tilde{U}$ respectively (which follows from their definitions). Therefore, the dominating singularity (which is also the radius of convergence) of $T$ occurs at the point $z=z_{0}$ for which

$$
z_{0} \exp \left(H_{1}\left(z_{0}\right)\right)=\frac{1}{e}
$$

see [6]; the numerical value of $z_{0}$ is 0.338321856899 . Since $z \exp \left(H_{1}(z)\right)$ is a power series with nonnegative coefficients (of which the coefficient of $z^{1}$ is strictly positive), $z_{0}$ is the unique solution on the circle of convergence of $T$. Similarly, the dominating singularity $\tilde{z}_{0}$ of $\tilde{U}$ is the solution of the equation

$$
\tilde{z}_{0} \exp \left(H_{2}\left(\tilde{z}_{0}\right)\right)=\frac{1}{e}
$$

Trivially, $H_{2}(z) \unlhd H_{1}(z)$ (since $U(z) \unlhd T(z)$ by definition), and by Lemma 2 , the inequality is strict for at least one coefficient. Therefore, $H_{2}(z)<H_{1}(z)$ for all $z>0$ for which both series converge. This implies immediately that $\tilde{z}_{0}>z_{0}$, i.e. $\tilde{U}$ has a larger radius of convergence than $T$. Therefore,

$$
\left[z^{n}\right] U(z) \leq\left[z^{n}\right] \tilde{U}(z) \ll\left(\frac{z_{0}}{\tilde{z}_{0}}+\epsilon\right)^{n}\left[z^{n}\right] T(z)
$$

for every $\epsilon>0$. In other words, the proportion of rooted trees in $\mathcal{R}_{n} \backslash \mathcal{R}_{n}^{(m)}(0,0)$ among all rooted trees in $\mathcal{R}_{n}$ is $O\left(\left(\frac{z_{0}}{\tilde{z}_{0}}+\epsilon\right)^{n}\right)$. Since every tree on $n$ vertices has at least one and at most $n$ representations as a rooted tree, the same statement holds if "rooted tree" is replaced by "tree". This proves Theorem 1 a fortiori $\left(\mathcal{R}_{n}^{(m)}(0,0)\right.$ is a subset of the set of all trees in $\mathcal{R}_{n}$ whose number of independent sets is divisible by $m$ ) with $c=\frac{z_{0}}{\tilde{z}_{0}}+\epsilon<1$ for suitable $\epsilon$. 


\section{The special case $m=2$}

In this section, everything will be made explicit in the case $m=2$. In this case, there are only four different sets $\mathcal{R}(i, j)=\mathcal{R}^{(2)}(i, j) \quad(0 \leq i, j \leq 1$; in the following, we will always drop the superscript for convenience), so that four different auxiliary generating functions (one for each of the sets) will be necessary. Let us denote these generating functions by $T_{i j}(z), 0 \leq i, j \leq 1$. It follows easily from (1) that

- a tree belongs to $\mathcal{R}(0,0)$ if and only if at least one of its subtrees belongs to $\mathcal{R}(0,0)$ or if one of its subtrees belongs to $\mathcal{R}(0,1)$ and another subtree to $\mathcal{R}(1,0)$,

- a tree belongs to $\mathcal{R}(0,1)$ if and only if all its subtrees belong to $\mathcal{R}(1,1)$,

- a tree belongs to $\mathcal{R}(1,0)$ if and only if all its subtrees belong to $\mathcal{R}(0,1) \cup \mathcal{R}(1,1)$, but not all of them to $\mathcal{R}(1,1)$,

- a tree belongs to $\mathcal{R}(1,1)$ if and only if all its subtrees belong to $\mathcal{R}(1,0) \cup \mathcal{R}(1,1)$, but not all of them to $\mathcal{R}(1,1)$.

This can be translated to the world of generating functions as follows:

$$
\begin{aligned}
& T_{01}(z)=z \exp \left(\sum_{n \geq 1} \frac{1}{n} T_{11}\left(z^{n}\right)\right), \\
& T_{10}(z)=z \exp \left(\sum_{n \geq 1} \frac{1}{n}\left(T_{01}\left(z^{n}\right)+T_{11}\left(z^{n}\right)\right)\right)-T_{01}(z), \\
& T_{11}(z)=z \exp \left(\sum_{n \geq 1} \frac{1}{n}\left(T_{10}\left(z^{n}\right)+T_{11}\left(z^{n}\right)\right)\right)-T_{01}(z), \\
& T_{00}(z)=T(z)-T_{01}(z)-T_{10}(z)-T_{11}(z),
\end{aligned}
$$

where $T(z)$ is the generating function for all rooted trees. From these equations, we can determine the first few coefficients of the generating functions for rooted trees with an even resp. odd number of independent sets:

$$
\begin{aligned}
& T_{00}(z)+T_{01}(z)=z+2 z^{4}+4 z^{5}+10 z^{6}+29 z^{7}+74 z^{8}+191 z^{9}+519 z^{10}+\ldots \\
& T_{10}(z)+T_{11}(z)=z^{2}+2 z^{3}+2 z^{4}+5 z^{5}+10 z^{6}+19 z^{7}+41 z^{8}+95 z^{9}+200 z^{10}+\ldots
\end{aligned}
$$

We focus on the first three equations, which we rewrite as

$$
\begin{aligned}
& T_{01}(z)=z \exp \left(T_{11}(z)+H_{1}(z)\right) \\
& T_{10}(z)=z \exp \left(T_{01}(z)+T_{11}(z)+H_{2}(z)\right)-T_{01}(z), \\
& T_{11}(z)=z \exp \left(T_{10}(z)+T_{11}(z)+H_{3}(z)\right)-T_{01}(z)
\end{aligned}
$$

for suitable auxiliary functions $H_{1}, H_{2}, H_{3}$. It is clear from these equations that $T_{01}$, $T_{10}$ and $T_{11}$ have the same dominating singularity $\rho$. By their definitions, the radius of 
convergence of $H_{1}, H_{2}, H_{3}$ is larger than $\rho$. At the dominating singularity, the Jacobian determinant of this system of functional equations has to vanish (otherwise, $T_{01}, T_{10}, T_{11}$ would have analytic continuations by the implicit function theorem). In other words,

$$
\begin{aligned}
& \left|\begin{array}{ccc}
1 & 0 & -z e^{T_{11}(z)+H_{1}(z)} \\
1-z e^{T_{01}(z)+T_{11}(z)+H_{2}(z)} & 1 & -z e^{T_{01}(z)+T_{11}(z)+H_{2}(z)} \\
1 & -z e^{T_{10}(z)+T_{11}(z)+H_{3}(z)} & 1-z e^{T_{10}(z)+T_{11}(z)+H_{3}(z)}
\end{array}\right| \\
& =\left|\begin{array}{ccc}
1 & 0 & -T_{01}(z) \\
1-T_{10}(z)-T_{01}(z) & 1 & -T_{10}(z)-T_{01}(z) \\
1 & -T_{01}(z)-T_{11}(z) & 1-T_{01}(z)-T_{11}(z)
\end{array}\right| \\
& =1-T_{01}(z)^{3}-T_{01}(z) T_{10}(z)-T_{01}(z)^{2} T_{10}(z)-T_{11}(z)-T_{01}(z)^{2} T_{11}(z) \\
& -T_{10}(z) T_{11}(z)-T_{01}(z) T_{10}(z) T_{11}(z)=0
\end{aligned}
$$

at $z=\rho$. Together with the three functional equations, this yields a system of three equations for $\rho, T_{01}(\rho), T_{10}(\rho)$ and $T_{11}(\rho)$ that can be solved numerically (the coefficients of the auxiliary functions $H_{1}, H_{2}, H_{3}$ are determined up to $z^{100}$, say, which provides an excellent approximation, since the radius of convergence of $H_{1}, H_{2}, H_{3}$ is larger than that of $\left.T_{01}, T_{10}, T_{11}\right)$. This yields a numerical value of $\rho=0.383108019849$ for the singularity.

Now $T_{01}, T_{10}, T_{11}$ can be expanded around $z=\rho$ (cf. $\left.[6,7]\right)$ :

$$
\begin{aligned}
& T_{01}(z)=a_{1}-b_{1} \sqrt{\rho-z}+c_{1}(\rho-z)+\ldots, \\
& T_{10}(z)=a_{2}-b_{2} \sqrt{\rho-z}+c_{2}(\rho-z)+\ldots, \\
& T_{11}(z)=a_{3}-b_{3} \sqrt{\rho-z}+c_{3}(\rho-z)+\ldots,
\end{aligned}
$$

which leads to an asymptotic formula for the number of rooted trees on $n$ vertices whose number of independent sets is odd by means of singularity analysis (see for instance [6]): this number is asymptotically equal to

$$
\frac{b_{2}+b_{3}}{2 \sqrt{\pi}} \cdot n^{-3 / 2} \cdot \rho^{-n+1 / 2}=(0.421841735367) \cdot n^{-3 / 2} \cdot(2.610229878230)^{n} .
$$

If one is interested in the number of trees rather than the number of rooted trees, one has to apply Otter's theorem $[7,18]$ that states that the number of representations of a tree as a rooted tree equals one plus the number of representations as an edge-rooted tree (an edge-rooted tree can be regarded as two rooted trees, joined at their roots by an edge). In the case of trees without restrictions, this yields the generating function

$$
T(z)-\frac{1}{2}\left(T(z)^{2}-T\left(z^{2}\right)\right)
$$

for the number of trees on a given number of vertices, where $T(z)$ is again the generating function for rooted trees. For our problem, we only have to determine when a tree that is constructed by joining two rooted trees at their roots has an odd number of independent 
sets (given the parities of $i$ and $i_{0}$ for both trees), which is not difficult. The result is the following generating function for trees with an odd number of independent sets:

$$
\begin{aligned}
S(z)= & T_{10}(z)+T_{11}(z)-T_{01}(z) T_{10}(z)-T_{10}(z) T_{11}(z) \\
& \quad-\frac{1}{2}\left(T_{01}(z)^{2}+T_{11}(z)^{2}-T_{01}\left(z^{2}\right)-T_{11}\left(z^{2}\right)\right) \\
= & z^{2}+z^{3}+z^{4}+2 z^{5}+3 z^{6}+5 z^{7}+9 z^{8}+17 z^{9}+32 z^{10}+\ldots
\end{aligned}
$$

The expansion of $S(z)$ around the dominating singularity is given by

$$
a_{4}+c_{4}(\rho-z)+d_{4}(\rho-z)^{3 / 2}+\ldots ;
$$

it can be deduced from the equations above that the coefficient of $\sqrt{\rho-z}$ vanishes. This is a general phenomenon, see [8]. It follows that the number of trees on $n$ vertices and an odd number of independent sets is asymptotically

$$
\frac{3 d_{4}}{4 \sqrt{\pi}} \cdot n^{-5 / 2} \cdot \rho^{-n+3 / 2}=(0.524015437477) \cdot n^{-5 / 2} \cdot(2.610229878230)^{n} .
$$

Therefore, one can finally deduce that the proportion of trees with an odd number of independent sets is $\sim(0.958896816978) \cdot(0.883097819337)^{n}$ among rooted trees on $n$ vertices and $\sim(0.979560376268) \cdot(0.883097819337)^{n}$ among (unrooted) trees on $n$ vertices. Here we make use of the known asymptotic formulæ for the number of rooted trees resp. trees on $n$ vertices. Table 1 compares the numbers for $n \leq 20$.

\section{Conclusion}

Similar results can be obtained for related parameters as well; for instance, if one considers the number of independent edge subsets (matchings) rather than that of independent vertex subsets, one obtains the following recursive relations in place of (1):

$$
e(T)=\sum_{i=1}^{k} e_{0}\left(T_{i}\right) \prod_{\substack{j=1 \\ j \neq i}}^{k} e\left(T_{j}\right)+\prod_{j=1}^{k} e\left(T_{j}\right), \quad e_{0}(T)=\prod_{j=1}^{k} e\left(T_{j}\right)
$$

Here, $e(T)$ denotes the number of independent edge subsets of a rooted tree $T$, and $e_{0}(T)$ the number of those independent edge subsets that do not cover the root. It is not difficult to see that the entire proof can be carried out along the same lines to show that the proportion of those trees for which the number of independent edge subsets is divisible by $m$ tends to 1 for any fixed $m$ as the number of vertices increases.

As mentioned in the introduction, an open conjecture states that all but finitely many positive integers are the number of independent sets of some tree. At this stage, it is not even clear that all residue classes modulo $m$ are covered for some fixed modulus $m$. We state this as a somewhat weaker conjecture: 


\begin{tabular}{|c||c|c|c|c|}
\hline \multicolumn{1}{|c||}{$\begin{array}{c}\text { number of } \\
\text { vertices }\end{array}$} & \multicolumn{2}{c|}{ Rooted trees } & \multicolumn{2}{c|}{ Trees } \\
\cline { 2 - 5 } & even & odd & even & odd \\
\hline 1 & 1 & 0 & 1 & 0 \\
2 & 0 & 1 & 0 & 1 \\
3 & 0 & 2 & 0 & 1 \\
4 & 2 & 2 & 1 & 1 \\
5 & 4 & 5 & 1 & 2 \\
6 & 10 & 10 & 3 & 3 \\
7 & 29 & 19 & 6 & 5 \\
8 & 74 & 41 & 14 & 9 \\
9 & 191 & 95 & 30 & 17 \\
10 & 519 & 200 & 74 & 32 \\
11 & 1387 & 455 & 172 & 63 \\
12 & 3711 & 1055 & 421 & 130 \\
13 & 10078 & 2408 & 1033 & 268 \\
14 & 27371 & 5602 & 2589 & 570 \\
15 & 74560 & 13251 & 6510 & 1231 \\
16 & 204167 & 31214 & 16626 & 2694 \\
17 & 560606 & 74241 & 42671 & 5958 \\
18 & 1543188 & 177971 & 110492 & 13375 \\
19 & 4261175 & 427501 & 287745 & 30210 \\
20 & 11794516 & 1031712 & 754192 & 68873 \\
\hline
\end{tabular}

Table 1: Number of rooted trees/trees with an even/odd number of independent sets

Conjecture 1 For all positive integers $m$ and $k$, there exists a tree $T$ such that

$$
i(T) \equiv k \bmod m \text {. }
$$

As also mentioned in the introduction, it would be interesting to know whether the number of independent sets of a tree has a limiting distribution. While a local limit theorem (see [6, Chapter 9] for an extensive treatment of various central and local limit laws) is clearly out of the question in view of the result of this paper, it is still conceivable that there might be a central limit theorem for this parameter.

\section{References}

[1] W. Y. C. Chen, L. W. Shapiro, and L. L. M. Yang. Parity reversing involutions on plane trees and 2-Motzkin paths. European J. Combin., 27(2):283-289, 2006.

[2] R. M. Corless, G. H. Gonnet, D. E. G. Hare, D. J. Jeffrey, and D. E. Knuth. On the Lambert $W$ function. Adv. Comput. Math., 5(4):329-359, 1996.

[3] M. Drmota. Asymptotic distributions and a multivariate Darboux method in enumeration problems. J. Combin. Theory Ser. A, 67(2):169-184, 1994. 
[4] S.-P. Eu, S.-C. Liu, and Y.-N. Yeh. Odd or even on plane trees. Discrete Math., 281(1-3):189-196, 2004.

[5] P. Flajolet, Z. Gao, A. Odlyzko, and B. Richmond. The distribution of heights of binary trees and other simple trees. Combin. Probab. Comput., 2(2):145-156, 1993.

[6] P. Flajolet and R. Sedgewick. Analytic Combinatorics. Cambridge Univ. Press, 2009.

[7] F. Harary and E. M. Palmer. Graphical enumeration. Academic Press, New York, 1973.

[8] F. Harary, R. W. Robinson, and A. J. Schwenk. Twenty-step algorithm for determining the asymptotic number of trees of various species. J. Austral. Math. Soc. Ser. A, 20(4):483-503, 1975.

[9] C. Heuberger and S. G. Wagner. Maximizing the number of independent subsets over trees with bounded degree. J. Graph Theory, 58(1):49-68, 2008.

[10] S. Janson. The Wiener index of simply generated random trees. Random Structures Algorithms, 22(4):337-358, 2003.

[11] P. Kirschenhofer, H. Prodinger, and R. F. Tichy. Fibonacci numbers of graphs. II. Fibonacci Quart., 21(3):219-229, 1983.

[12] P. Kirschenhofer, H. Prodinger, and R. F. Tichy. Fibonacci numbers of graphs. III. Planted plane trees. In Fibonacci numbers and their applications (Patras, 1984), volume 28 of Math. Appl., pages 105-120. Reidel, Dordrecht, 1986.

[13] X. Li, Z. Li, and L. Wang. The inverse problems for some topological indices in combinatorial chemistry. J. Comput. Biol., 10:47-55, 2003.

[14] X. Li, H. Zhao, and I. Gutman. On the Merrifield-Simmons index of trees. MATCH Commun. Math. Comput. Chem., 54(2):389-402, 2005.

[15] S. B. Lin and C. Lin. Trees and forests with large and small independent indices. Chinese J. Math., 23(3):199-210, 1995.

[16] V. Linek. Bipartite graphs can have any number of independent sets. Discrete Math., 76(2):131-136, 1989.

[17] R. E. Merrifield and H. E. Simmons. Topological Methods in Chemistry. Wiley, New York, 1989.

[18] R. Otter. The number of trees. Ann. of Math. (2), 49:583-599, 1948.

[19] A. S. Pedersen and P. D. Vestergaard. The number of independent sets in unicyclic graphs. Discrete Appl. Math., 152(1-3):246-256, 2005.

[20] A. S. Pedersen and P. D. Vestergaard. An upper bound on the number of independent sets in a tree. Ars Combin., 84:85-96, 2007.

[21] H. Prodinger and R. F. Tichy. Fibonacci numbers of graphs. Fibonacci Quart., 20(1):16-21, 1982.

[22] S. Wagner and H. Wang. On the parity of the Wiener index. European J. Combin., 30(4):996-1004, 2009.

[23] A. Yu and X. Lv. The Merrifield-Simmons indices and Hosoya indices of trees with $k$ pendant vertices. J. Math. Chem., 41(1):33-43, 2007. 